\title{
EDITORIAL
}

\section{Mixed bag}

So, you'll notice an exciting new feature called Today's 2 signs (edited and managed by Ami Bajwa, a new addition to the editorial team). This is supposed to teach us some solid signs 'by great example' or some unusual and new signs... What a great idea!

You will also note the emphasis of the review articles and Specialty Corner on PACS. These are simple, beginners' guides to PACS written by Otto Schulze, who while still a registrar in radiology, leads the whole Western Cape PACS vision. It's guys like Otto who have both IT and radiology insight, who help us understand and make better decisions. I hope that after completion of his thesis, also on radiology system and information that Otto will lead the way for a new area of radiology specialisation in South Africa (IT in radiology is an existing following option in the USA).

It is also time we brought to attention our imaging libraries and to liven the discussion around these. Should the historical hard-copy libraries be preserved? Should these be digitised? Should we incorporate the library cases by demarcating them on our PACS? Are we duplicating work already done, for example by AMIRSYS (Anne Osborne \& Ric Harnsberger), a new digital library source available on line. In a future issue, I will give you a clearer idea on 'STAT Dx' as we will get free access to this (expensive, but super) resource which was demonstrated to me in Utah.

Lastly, just a word on the scholarship that I have completed. Let me encourage you to apply. The award was $\$ 10000$ for $2-3$ months at one of any of the recommended sites including Ivy League universities, such as Columbia in New York and Harvard in Boston.

I did predominantly 'Diffusion Tenor Imaging (DIT)', started a number of collaborative research projects and got myself appointed as a Research Scientist at Columbia for the next year. I hope this is the start of a long fruitful association. I will give a full report in the next issue. I hope you enjoy this issue because it's full of wonderful bits of information!

\section{Savvas Andronikou}

Chief Editor 